\title{
Biomarkers and personalised medicine for asthma
}

\author{
Michael Roth ${ }^{1,2}$ and Daiana Stolz ${ }^{1,2}$ \\ Affiliations: ${ }^{1}$ Pulmonary Care Division, Internal Medicine, University Hospital Basel, Basel, Switzerland. \\ ${ }^{2}$ Pulmonary Cell Research, Dept Biomedicine, University Basel, Basel, Switzerland.
}

Correspondence: Daiana Stolz, University Hospital Basel, Pulmonary Care Division, Petersgraben 4, Basel, 4031, Switzerland. E-mail: Daiana.stolzQusb.ch

@ERSpublications

Precision medicine and personalised therapy are major goals for chronic inflammatory diseases such as asthma. These two aims can only be achieved when the different subtypes of asthma can be precisely defined by robust diagnostic criteria. http://ow.ly/2rsy30mODVS

Cite this article as: Roth M, Stolz D. Biomarkers and personalised medicine for asthma. Eur Respir J 2019; 53: 1802094 [https://doi.org/10.1183/13993003.02094-2018].

Asthma is the most common chronic lung disease, affecting approximately 300 million people worldwide. Its prevalence has increased over the past 40 years [1]. Symptoms can be controlled by anti-inflammatory drugs and bronchodilators but, so far, the disease cannot be cured $[1,2]$.

The Global Initiative for Asthma (GINA) has defined asthma as a heterogeneous condition that presents with different phenotypes, varying according to the patient's history, age of symptom onset, presence of allergies, eosinophil counts, and severity. In addition, asthma phenotypes have been linked to corticosteroid response and long-term patient outcomes [3-5]. Until recently, the progress in treating asthma has been slow, perhaps because treatment was mostly based on clinical measures of disease severity, rather than on the underlying pathogenetic mechanism [6]. Although clinical parameters are useful, they provide only limited information regarding asthma phenotypes. A better subgrouping of asthma pheno/endotypes could be potentially achieved by combining clinical parameters with transcriptome expression profiles. Pheno/endotype subgrouping by combined clinical and cell biological characteristics may explain increased exacerbation risks, and provide the basis for personalised therapies. Understanding the contribution of these parameters to the pathogenesis of asthma may answer the question of whether asthma is a single disease, or if it encapsulates different diseases that are presented in the clinic as asthma [3-5].

The most recent definition of asthma phenotypes, dichotomising asthma according to the level of CD4+ type 2 helper cells and their cytokines, were assigned as T2-high and T2-low endotypes. However, the information on biomarkers that distinguish certain asthma pheno/endotypes and their specific response to therapy is still insufficient $[2,3]$. Thus, further research is required to establish asthma subgroups based on combined expression patterns of specific pro-inflammatory cytokines, cell surface molecules, cellular signalling pathways, genetic pre-disposition, epi-genomics, and asthma risk factors [3-5].

The assessment of transcriptomic patterns combined with clinical parameters presented in the current issue of the European Respiratory Journal by PAVLIDIs et al. [7], is a step forward improving phenotyping and endotyping of asthma. The study evaluated both clinical characteristics, including age of onset, gender, patient history, comorbidities and lifestyle, and the biomarkers periostin, chloride channel accessory 1 (CLCA1) and serpin $\beta 2$ to subtype 103 asthmatics into T2-high and T2-low endotypes. In contrast to 
other studies [5, 8], CLCA1 was not expressed by epithelial cells and was therefore excluded from the analysis. Yet, the lack of CLCA1 may have been affected by the different lifestyle or environmental conditions of the study cohort [9]. PAVLIDIs et al. [7] confirmed, however, that high eosinophil counts and increased levels of exhaled nitric oxide are associated with the T2-high endotype. Conversely, and conflicting with previous data, IgE, periostin and serpin $\beta 2$ did not help to identify the T2-high endotype $[4,5,8]$. It was somewhat surprising that the better characterisation of the underlying immune response by the addition of these three biomarkers did not change the subgrouping compared to the grouping based on clinical parameters alone. The study, however, highlights the urgent need to find better indicators for asthma pheno/endotypes and indicates that subgrouping of asthma may have to be re-considered.

Earlier studies subgrouped asthma in T1-T4 endotypes based on the patient's history and showed that eosinophil counts separated the T1 subtype from the other groups, while no differences were observed for exhaled nitric oxide, serum IgE or neutrophil counts [9]. Genetic predisposition to asthma has been discussed for years, but has failed to provide any solution to the problem [10]. Genome-wide association studies were combined with lung eQTL analysis and suggested three novel genes (ABI3BP, NAF1 and MICA) to be involved in the pathogenesis of asthma; all others had been indicated by earlier studies [11]. Further analysis showed that most of the new genes for asthma susceptibility were regulators of well-known asthma-relevant cytokines, such as IL-33 [11]. Therefore, other phenotyping systems including the function of muscle or epithelial cells should also be considered [12].

The major problem regarding asthma pheno/endotyping is the definition of the parameters, symptoms and biomarkers to indicate a specific asthma phenotype. Furthermore, it is unclear how steady asthma phenotypes are. It has been suggested that, under certain conditions, gender might affect the phenotype of asthma patients [13]. Several questions remain without an answer. Does the short-term effect of risk factors modify asthma phenotypes? Does the circadian rhythm affect parameters that characterise asthma phenotypes, as was described for COPD and other chronic inflammatory disease [14, 15]? Furthermore, there is an unmet need for studies that determine if a specific marker is an indicator for the progression of the disease or if it is a consequence of the disease. In general, the application of omics (genomics, transcriptomics, proteomics, etc.) has yet to yield usable biomarkers for asthma or any other chronic inflammatory lung disease. Thus, it may be necessary to reconsider the pathogenesis and pathologies of asthma $[1,3]$.

Precision medicine and personalised therapy are major goals for chronic inflammatory diseases such as asthma [16-18]. These two aims can only be achieved when the different subtypes of asthma can be precisely defined by robust diagnostic criteria, including genetic markers and biomarkers.

Conflict of interest: M. Roth has nothing to disclose. D. Stolz reports consultancy fees from AstraZeneca and Novartis, and grants from AstraZeneca, Pan Gas, Weimann and Curetis, outside the submitted work.

\section{References}

$1 \quad$ Papi A, Brightling C, Pedersen SE, et al. Asthma. Lancet 2018; 391: 783-800.

2 Chung KF. Precision medicine in asthma: linking phenotypes to targeted treatments. Curr Opin Pulm Med 2018; 24: $4-10$.

3 Matucci A, Vultaggio A, Maggi E, et al. Is IgE or eosinophils the key player in allergic asthma pathogenesis? Are we asking the right question? Respir Res 2018; 19: 113.

4 Woodruff PG, Modrek B, Choy DF, et al. T-helper type 2-driven inflammation defines major subphenotypes of asthma. Am J Respir Crit Care Med 2009; 180: 388-395.

5 Woodruff PG, Boushey HA, Dolganov GM, et al. Genome-wide profiling identifies epithelial cell genes associated with asthma and with treatment response to corticosteroids. Proc Natl Acad Sci USA 2007; 104: 15858-15863.

6 Fahy JV. Type 2 inflammation in asthma-present in most, absent in many. Nat Rev Immunol 2015; 15: 57-65.

7 Pavlidis S, Takahashi K, Ng Kee Kwong F, et al. "T2-high" in severe asthma related to blood eosinophil, exhaled nitric oxide and serum periostin. Eur Respir J 2019; 53: 1800938.

8 Fang F, Pan J, Li Y, et al. Identification of potential transcriptomic markers in developing asthma: An integrative analysis of gene expression profiles. Mol Immunol 2017; 92: 38-44.

9 Lefaudeux D, De Meulder B, Loza MJ, et al. U-BIOPRED clinical adult asthma clusters linked to a subset of sputum omics. J Allergy Clin Immunol 2017; 139: 1797-1807.

10 Meyers DA, Bleecker ER, Holloway JW, et al. Asthma genetics and personalised medicine. Lancet Respir Med 2014; 2: 405-415.

11 Nieuwenhuis MA, Siedlinski M, van den Berge M, et al. Combining genomewide association study and lung eQTL analysis provides evidence for novel genes associated with asthma. Allergy 2016; 71: 1712-1720.

12 An SS, Mitzner W, Tang WY, et al. An inflammation-independent contraction mechanophenotype of airway smooth muscle in asthma. J Allergy Clin Immunol 2016; 138: 294-297.

13 Tse SM, Rifas-Shiman SL, Coull BA, et al. Sex-specific risk factors for childhood wheeze and longitudinal phenotypes of wheeze. J Allergy Clin Immunol 2016; 138: 1561-1568.

14 Canonica GW, Blasi F, Scichilone N, et al. Characterization of circadian COPD symptoms by phenotype: methodology of the STORICO observational study. Eur J Intern Med 2017; 43: 62-68. 
15 Dashti HS, Follis JL, Smith CE, et al. Gene-environment interactions of circadian-related genes for cardiometabolic traits. Diabetes Care 2015; 38: 1456-1466.

16 Garcia-Marcos L, Edwards J, Kennington E, et al. Priorities for future research into asthma diagnostic tools: A PAN-EU consensus exercise from the European asthma research innovation partnership (EARIP). Clin Exp Allergy 2018; 48: 104-120.

17 Svenningsen S, Nair P. Asthma endotypes and an overview of targeted therapy for asthma. Front Med (Lausanne) 2017; 4: 158

18 Canonica GW, Ferrando M, Baiardini I, et al. Asthma: personalized and precision medicine. Curr Opin Allergy Clin Immunol 2018; 18: 51-58. 